\title{
Neurocomputational mechanisms of reinforcement-guided learning in humans: A review
}

\author{
Michael X CoHen \\ University of California, Davis, California \\ and University of Bonn, Bonn, Germany
}

\begin{abstract}
Adapting decision making according to dynamic and probabilistic changes in action-reward contingencies is critical for survival in a competitive and resource-limited world. Much research has focused on elucidating the neural systems and computations that underlie how the brain identifies whether the consequences of actions are relatively good or bad. In contrast, less empirical research has focused on the mechanisms by which reinforcements might be used to guide decision making. Here, I review recent studies in which an attempt to bridge this gap has been made by characterizing how humans use reward information to guide and optimize decision making. Regions that have been implicated in reinforcement processing, including the striatum, orbitofrontal cortex, and anterior cingulate, also seem to mediate how reinforcements are used to adjust subsequent decision making. This research provides insights into why the brain devotes resources to evaluating reinforcements and suggests a direction for future research, from studying the mechanisms of reinforcement processing to studying the mechanisms of reinforcement learning.
\end{abstract}

Our world is filled with uncertainty and limited resources. Nearly every decision we face is clouded in uncertainty - uncertainty about the consequences of our decisions, uncertainty about whether others will obtain resources before we do, uncertainty about how different individuals will respond in similar contexts. Fortunately, we are often faced with the same or similar decision problems repeatedly, providing the opportunity to learn from our previous decisions and outcomes and to dynamically adapt decision-making strategies. The ability to use reinforcements flexibly to modify goal-directed behavior in the service of maximizing rewards and minimizing punishments is termed reinforcement learning. Theories and computational models of reinforcement learning provide a biologically and mathematically grounded framework within which to characterize, predict, and understand the behavioral, cognitive, and neural bases of reward-guided decision making. The brain is highly adept at reinforcement learning, and although the neural processes that support this adaptation are being discovered, much remains unknown about the mechanisms by which rewards and punishments are used by the brain to optimize and guide decision making. Recent research in neuroscience and computational modeling suggests that theories of reinforcement learning provide a useful framework within which to study the behavioral and neural mechanisms of reward-based learning and decision making (Bayer \& Glimcher, 2005; Camerer, 2003; Dayan \& Balleine,
2002; Egelman, Person, \& Montague, 1998; Montague \& Berns, 2002; O’Doherty, Hampton, \& Kim, 2007; Schultz, Dayan, \& Montague, 1997). This literature seems to be in its infancy and yet is making noteworthy strides in characterizing the brain systems and neural computations that support reinforcement learning.

Imaging and single-unit recording studies have demonstrated that reinforcements elicit increases in neural activity in several brain regions - notably, those in midbrain-striatal-frontal cortical circuits. Dopamine appears to be critically involved in such processes, although its precise function is still debated (Berridge, 2007; Montague, Hyman, \& Cohen, 2004; Redgrave \& Gurney, 2006; Schultz, 1998). Generally, changes in brain activity strongly correlate with reinforcements and their predictors, with some regions exhibiting increases in activity following rewards, as compared with losses, and some regions exhibiting increases in activity following losses, as compared with rewards. One might ask the simple question, why? Why should these brain circuits "care about" reinforcements? If the reinforcement already occurred, why continue processing and evaluating its relative reward value after it has been consumed or received? The straightforward and intuitive answer is that reinforcements are useful for guiding future decisions and actions. That is, reinforcements provide information that can be used to adjust behavior to maximize future rewards when similar conditions or decision problems arise. Most of the neuroscience studies of reinforcement processing have

M. X Cohen, mikexcohen@gmail.com 
focused on elucidating the neural and neurochemical systems that are engaged during and immediately following reinforcements and their predictors; surprisingly little research has been conducted to investigate how reinforcements might be used to guide future behavior and decision making. This is surprising because of the seemingly obvious purpose of devoting brain resources to evaluating reinforcements - to use them to adapt future behavior-but it is also surprising because mathematically grounded theories used to characterize learning make simple, strong, and empirically testable predictions for how the brain might use reinforcements to guide decision making. Indeed, as will be discussed below, computational models often include mechanisms for using reinforcements to adapt future behavior. However, many of these models are based on abstract mathematical equations that are designed to enable the model to optimize its behavior; they are not designed bottom-up, from neurophysiology and neuroanatomy (although some recent models are increasingly constraining model architecture according to neurobiology, as will be discussed more below). In contrast, there is less empirical research that provides evidence for how the brain may utilize reinforcements to guide decision making. It is important to distinguish the empirical neuroscience research from the theoretical modeling work, because biological organisms and computational models may perform the same tasks using different mechanisms.

Here, I review the basic computational mechanisms that have been proposed to underlie the dynamic adjustment of behavior according to reinforcements, the predictions made by that class of models, and empirical tests inspired by those models that provide insight into the neural mechanisms of reinforcement learning and decision making. This review will focus on the functions of the medial frontal cortex, although other key structures will be discussed as well. The studies reviewed here do not include all the cognitive and neuroscientific experiments that relate rewards and punishments to behavioral performance; instead, the studies reviewed here are among those that have used reinforcement learning models to inspire and test predictions regarding the possible neurocomputational mechanisms that link the processing of rewards and losses to adjustments in subsequent decision making. Similarly, in many studies, changes in brain activity have been related to predictions from computational models, but only those studies that have specifically addressed the mechanisms by which reinforcements may be used to guide decision making will be given extensive discussion. Readers are referred elsewhere for more general overviews of the neural systems involved in decision making (Cardinal, 2006; Glimcher, Dorris, \& Bayer, 2005; Krawczyk, 2002) and more detailed analyses of computational models of learning and their proposed biological and neurochemical bases (Cardinal, 2006; O'Doherty et al., 2007; Pennartz, 1995).

\section{Computational Mechanisms of Reinforcement Learning}

Many computational models of reinforcement-guided learning exist. Each has its own benefits and limitations, and different models are more or less suited for different circumstances, depending on the particular assumptions made and the data or phenomena for which the model is designed to account. However, many reinforcement-learning models rely on similar mechanisms and have similar core features. In this section, I will review the basic features of reinforcement-learning models, without detailing all possible versions of the models and their extensions. The purpose is to provide a background for understanding and evaluating the empirical research that incorporates or is based on these models, especially research aimed at elucidating the neurocognitive mechanisms by which reinforcements guide decision making. Extensive reviews and comparisons of reinforcement-learning models can be found elsewhere (Sutton \& Barto, 1998). The basic learning mechanism behind many reinforcement-learning models can be summarized semantically by Thorndike's (1911) law of effect:

Of several responses made to the same situation, those which are accompanied or closely followed by satisfaction to the animal will, other things being equal, be more firmly connected with the situation, so that, when it recurs, they will be more likely to recur; those which are accompanied or closely followed by discomfort to the animal will, other things being equal, have their connections with that situation weakened, so that, when it recurs, they will be less likely to occur. The greater the satisfaction or discomfort, the greater the strengthening or weakening of the bond. (p. 244)

Thorndike states that actions associated with rewards are more likely to be repeated, whereas actions associated with losses or punishments are less likely to be repeated. In the context of the computational models reviewed here, weights are numerical representations of the strength or preference for particular actions, decisions, or stimuli. (In some models, weights are called $Q$ values, but their function essentially is the same.) Weights can be interpreted as the expected reward for a particular action. Thus, weights with large positive values are preferred, weights with large negative values are avoided, and weights with values close to zero are not preferred or avoided. When an agent (e.g., a subject in an experiment) must choose one from a list of possible decisions or actions, the numerical values of these weights are compared, and the weight with the largest positive value is selected. Typically, this choice selection is passed through a probabilistic function, so that the weight with the highest value is not necessarily always chosen and, sometimes, other choices are selected. Probabilistic selection ensures that behavior retains some stochastic component, which is useful for exploring the environment and avoiding situations in which, for example, other decision options provide higher rewards but are not selected because the agent is stuck continually choosing one decision option.

In order to optimize behavior, weights must be adjusted according to reinforcements. This is done by an updating term, which can take several possible forms. The most commonly used updating term is called a reward prediction error and is the difference between a reward expected 
and a reward received: $d=r-w$, where $d$ is the prediction error, $r$ is the reward, and $w$ is the value of the weight corresponding to the action selected. Thus, when rewards that follow particular actions are greater than the reward expected from that particular action (i.e., the weight), the prediction error is positive; when rewards are received exactly as expected, the prediction error is zero; and when rewards are smaller than those expected, the prediction error is negative. These prediction errors can be used to adjust the weight: $w_{t}=d+w_{t-1}$, where $t$ refers to a trial. In this way, actions that lead to rewards (punishments) are strengthened (weakened) and become more (less) likely to be chosen in subsequent trials. Thus, $w_{t}=d+w_{t+1}$ can be seen as a concise mathematical representation of part of Thorndike's (1911) law of effect.

Typically, prediction error terms are multiplied by a learning rate, which scales the impact of the prediction error on the adjustment of the weight: $w_{t}=a * d+w_{t-1}$. Typically, $a$ is between 0 and 1, although values outside this range may be useful in some circumstances. The learning rate describes how the prediction error adjusts the weights (i.e., the model's sensitivity to previous outcomes): With large learning rates, the prediction error makes large adjustments to future weights; with small learning rates, the prediction error has a smaller impact on the model's weights and decisions. At the extreme case of a learning rate of 0 , the model's weights and decision making are not influenced by prediction errors.

The advantage of using a prediction error term is that the agent needs no a priori knowledge of the reinforcement consequences of different decisions. It is also possible to use other adjustment parameters that are not based on calculating the difference between expected and received rewards. In these cases, the agent is not learning new decision-reward associations but, rather, is dynamically adjusting its behavior according to ongoing reinforcements. This updating has the general form $w_{t+1}=$ $a * l+w_{t}$, where $l$ is a parameter with a fixed value that is associated a priori with a particular reinforcement from a particular decision. In these cases, the agent must know the possible reinforcement consequences of each action. This situation is sometimes called an $n$-armed bandit problem (Sutton \& Barto, 1998). The difference between this learning parameter $(l)$ and the reward value $(r)$ is that the reward value is a function of the reinforcement, whereas this learning parameter can have a value separate from the rewarding properties of the reinforcement. For example, if a risk-averse individual wins a high-risk gamble, he might decide that the odds are against winning again; in this case, although winning the gamble resulted in a positive reward, the learning parameter may be negative because this reinforcement makes the individual less likely to gamble again (i.e., the reinforcement, although positive, decreases the weight of the decision to gamble). Thus, $r$ and $l$ can have the same value in some circumstances, but they can also be different.

These models are simplistic. They do not capture all aspects of decision making, especially during decisionmaking situations that require complex strategies. Nor do they account for individual differences in how different agents might use the same reinforcements in different ways. However, these models are robust across a range of species, circumstances, and experimental setups. As will be reviewed in later sections, these equations appear to capture some aspects of the processes engaged by the brain during reinforcement learning and decision making. These models therefore provide a mathematically and neurobiologically grounded framework within which to (1) generate novel hypotheses about how reinforcements might be used to guide or optimize decision making and (2) interpret results from empirical studies in the contexts of larger theoretical approaches and previous research that utilizes similar reinforcement-learning approaches.

\section{Comparison of Models}

The modeling approach described in the previous section illustrates the principles behind one class of reinforcement-learning models that has been widely used in psychology and cognitive neuroscience. The present review focuses on this type of model because much of the empirical research linking reinforcements to decisionmaking adjustments has used this class of models. However, other reinforcement-learning models exist, and different models may be more or less suitable for different situations. Some of these models are extensions of the reinforcement-learning models reviewed in the previous section. One widely used extension is called temporal differences and is a mechanism for linking rewarding events to actions or stimuli that precede and predict them (Montague, Dayan, \& Sejnowski, 1996; Suri, 2002; Sutton \& Barto, 1990). Others have proposed conceptually similar but more efficient computational mechanisms by which agents can learn to associate rewards with actions or stimuli that precede and predict them (Bogacz, McClure, Li, Cohen, \& Montague, 2007). It has also been proposed that there are two separable components to these models: a critic, which computes a prediction error on the basis of a comparison of expected and received reinforcements, and an actor, which receives the prediction error signal from the critic and uses this signal to modify behavior (Houk \& Wise, 1995; Joel, Niv, \& Ruppin, 2002; O’Doherty et al., 2004; Suri \& Schultz, 1999). Other extensions to the standard temporal difference learning models are designed to account for more complex decision-making behavior and accompanying brain activity. For example, Hampton, Bossaerts, and O'Doherty (2006) used a Bayesian hidden state Markov model in combination with a standard temporal difference model to demonstrate that human subjects incorporate higher order information about task states during a reversal task; predictions from this model were confirmed by patterns of activation in the ventral prefrontal cortex. Daw, O'Doherty, Dayan, Seymour, and Dolan (2006) presented a computational model that incorporated representations of exploring versus exploiting learned response-reward contingencies. This model was used to show that activation in the prefrontal and parietal cortices correlated with whether subjects chose to maintain known rewarding strategies (exploit) or search for other, possibly more rewarding strategies (explore). Other researchers have also examined how computational 
models might inform the exploration-exploitation tradeoff (J. D. Cohen, McClure, \& Yu, 2007), and it has been proposed that norepinephrine may play a role in this balance (Aston-Jones \& Cohen, 2005).

Other abstract mathematical models have been proposed to account for neural mechanisms of decision making. These models do not necessarily rely on principles of reinforcement learning and may be better suited for decision-making situations with no long-term response strategies. For example, one type of model proposes that decisions (e.g., sensory decisions such as the direction of coherent motion in a noisy environment) are made by accumulating evidence for and against each decision option, and that a decision is made once enough evidence for a hypothesis has reached a threshold (Ditterich, 2006; Gold \& Shadlen, 2002). These models are often referred to as drift diffusion models (Ratcliff, 2002). This type of model might be relevant to cases of reward-based decision making - for example, if evidence must be accumulated over time in order to decide which of several actions will yield the most reward (Bogacz, Brown, Moehlis, Holmes, \& Cohen, 2006). In these cases, reward value or probability may modulate the decision threshold (Simen, Cohen, \& Holmes, 2006).

Other, more recent models are taking steps toward biological realism and complexity (O'Reilly, 2006). Notably relevant to reinforcement learning are models by Frank and colleagues (Frank, 2005, 2006; Frank \& Claus, 2006). These models abstract many lower level neurobiological functions, such as the dynamics of ion channel gating, while explicitly modeling aspects of neural systems that arguably are more relevant to cognitive/psychological processes, such as the nature and strength of connections among brain regions, and temporal neural dynamics. These models incorporate separate nodes for different brain structures, such as the substantia nigra, striatum, subthalamic nucleus, and orbitofrontal cortex. Within these regions, the equations governing the activity of each of several cells are based on empirically demonstrated neural processes. Different classes of cells have also been functionally delineated, such as striatal cells that contain predominantly D1 versus D2 receptors, which lie in putatively functionally and anatomically distinct striatal-basal ganglia loops. These models simulate increases and decreases in dopamine activity to modulate the strength of connections between sensory inputs and motor outputs (Frank, 2005). These models have been shown to predict differences in learning from positive versus negative feedback that are modulated by dopamine levels - for example, from changes in medication status in Parkinson's patients (Frank, Seeberger, \& O’Reilly, 2004). Predictions from these models have also been shown to predict eventrelated potential (ERP) responses to errors and to performance feedback (Frank, Woroch, \& Curran, 2005).

\section{Neural Activity Correlates With Components of Computational Models}

Before discussing examples of how computational learning models have increased our understanding of how reinforcements guide decision making, I will review re- search linking some aspects of these models - prediction errors, weights, and learning rates - to neural activity. This research demonstrates that the models are appropriate for uncovering cognitive and neural processes, even though aspects of these computational models were not originally based on neurobiology.

Neural correlates of reward prediction errors. Reward prediction errors are thought to be signaled by the phasic activation of midbrain dopamine cells in the ventral tegmental area and substantia nigra, pars compacta. The phasic dopamine neuron response is a rapidly onsetting, short-lived burst of activity that induces dopamine release in widespread regions of the limbic system. It occurs under a range of behaviorally significant events, primarily those that involve rewards or neutral stimuli that predict rewards (unconditioned and conditioned reinforcers, respectively; Schultz, 1998, 2006). With striking similarity to the prediction error term from reinforcement-learning models, when an unexpected reward is received, dopamine neurons increase their firing rate; when expected rewards are received, dopamine neurons do not change their firing rate; and when expected rewards are not received, dopamine neurons decrease their firing rate. Increasing evidence from action potentials of individual midbrain dopamine neurons in animals supports this assertion (Bayer \& Glimcher, 2005; Daw \& Doya, 2006; Hollerman \& Schultz, 1998; Nakahara, Itoh, Kawagoe, Takikawa, \& Hikosaka, 2004; Schultz, 1998; Schultz et al., 1997). Detailed reviews of this evidence can be found elsewhere (Schultz, 1998, 2001, 2006; Schultz \& Dickinson, 2000).

The discovery that the activity of dopamine neurons bears a striking similarity to prediction error terms in computational theories of learning has inspired many researchers using functional magnetic resonance imaging (fMRI) and scalp-recorded ERPs to investigate neural correlates of reward prediction errors in humans. In fMRI studies, researchers have used computational reinforcement-learning models to estimate reward prediction errors that might be experienced on each trial. These estimates can then be entered into a regression to identify voxels in the brain in which activity correlates with prediction errors derived from the model. Significant correlations are often observed in the striatum and frontal cortex (Braver \& Brown, 2003; Haruno et al., 2004; McClure, Berns, \& Montague, 2003; O’Doherty, Dayan, Friston, Critchley, \& Dolan, 2003; O'Doherty et al., 2007; Tanaka et al., 2004) and are interpreted as reflecting reward prediction error signals that may have been signaled from the midbrain dopamine neurons and transmitted to other regions of the brain. It is not necessary to use modeling approaches to identify neural correlations of prediction errors; regions in which activity correlates with prediction errors can also be identified through experimental design (e.g., Abler, Walter, Erk, Kammerer, \& Spitzer, 2006; McClure et al., 2003; Rolls, McCabe, \& Redoute, 2008).

Prediction errors can be positive (better than expected) or negative (worse than expected), leading to the question of whether similar or different networks in the brain encode positive versus negative prediction errors. Unfortunately, the fMRI literature does not yield a consistent 
message on this issue. For example, in some studies, positive and negative prediction errors were associated with increased and decreased activity, respectively, in frontostriatal regions (M. X Cohen, 2007; McClure et al., 2003; O'Doherty, Dayan et al., 2003); Rodriguez, Aron, and Poldrack (2006) reported ventral striatal activity correlated with negative, but not positive, prediction errors during classification learning; Yacubian et al. (2006) found that ventral striatal activity correlated with positive prediction errors, whereas amygdala activity correlated with negative prediction errors; and Seymour, Daw, Dayan, Singer, and Dolan (2007) suggested that different subregions of the striatum are preferentially involved in positive versus negative prediction errors. Finally, with regard to imaging the midbrain - the purported origin of prediction error signals-Murray et al. (2008) reported increased midbrain activity for rewards, as compared with punishments, whereas Aron et al. (2004) reported increased midbrain activity for negative, as compared with positive, feedback. The single-unit studies in the monkey midbrain demonstrate increases and decreases in firing rates for positive and negative prediction errors, respectively (Bayer, Lau, \& Glimcher, 2007; Schultz et al., 1997). One possible way to reconcile these findings would be if different but spatially overlapping neural networks encode negative versus positive prediction errors and are differentially activated according to the particular task. Another possibility is that the actual neural computations engaged in these regions are more complex and task dependent than simple prediction errors, as defined by the models, but that these computations resemble prediction errors enough to be statistically identified in regression analyses.

In addition to the uncertainty about the encoding of negative versus positive prediction errors, there is also uncertainty about the precise regions in which activity correlates with prediction errors: Brain regions in which activity correlates with prediction errors have been reported in the ventral striatum (nucleus accumbens and/or putamen; Abler et al., 2006; Knutson \& Wimmer, 2007; McClure et al., 2003; O’Doherty et al., 2004; Rolls et al., 2008; Seymour et al., 2007; Seymour et al., 2004), the dorsal striatum (Delgado, Miller, Inati, \& Phelps, 2005; Filoteo et al., 2005; Haruno \& Kawato, 2006), regions of the prefrontal cortex, including orbitofrontal, ventrolateral, and dorsolateral (M. X Cohen, 2007; O'Doherty, Dayan, et al., 2003; Ramnani, Elliott, Athwal, \& Passingham, 2004; Rolls et al., 2008), the midbrain (Aron et al., 2004; Murray et al., 2008), and the cerebellum (O'Doherty, Dayan, et al., 2003). One possibility is that prediction errors are signaled to different networks in the brain depending on the task (i.e., how those prediction errors are used); another possibility is that anatomical differences across studies are related to intersubject and interstudy variability and statistical thresholding.

The Schultz et al. (1997) work on dopamine neurons in monkeys has also inspired research using scalp-recorded ERPs in humans. Here, researchers have identified ERP modulations called the error-related negativity (ERN) and the related feedback-related negativity (FRN) that have been hypothesized to reflect a neural reward predic- tion error signal (Holroyd \& Coles, 2002). The FRN is a relatively negative ERP deflection at frontocentral scalp sites around $200-400 \mathrm{msec}$ following negative, as compared with positive, feedback (Frank et al., 2005; Holroyd, Nieuwenhuis, Yeung, \& Cohen, 2003; Nieuwenhuis et al., 2002; Yasuda, Sato, Miyawaki, Kumano, \& Kuboki, 2004), and it reflects neural processes that have several characteristics of prediction errors (M. X Cohen, Elger, \& Ranganath, 2007; M. X Cohen \& Ranganath, 2007; Frank et al., 2005; Holroyd \& Coles, 2002, 2008; Nieuwenhuis, Holroyd, Mol, \& Coles, 2004; Ruchsow, Grothe, Spitzer, \& Kiefer, 2002; Schultz et al., 1997; Yasuda et al., 2004). However, not all the characteristics of the FRN conform to prediction errors. For example, prediction errors should be scaled by how expected or probable the reward/ punishment is. Although some studies have shown the magnitude of the FRN to correlate with the expectedness of a feedback (M. X Cohen et al., 2007; Holroyd et al., 2003; Yasuda et al., 2004), other studies have shown no relationship (Hajcak, Holroyd, Moser, \& Simons, 2005; Hajcak, Moser, Holroyd, \& Simons, 2007). It has been proposed that the FRN reflects the impact of a reward prediction error signal originating in the midbrain dopamine system, which is then used to adapt reward-seeking behavior (Holroyd \& Coles, 2002). In more recent work, Holroyd and colleagues have expanded this theory and the associated modeling work to better simulate behavioral and neural processes during both reinforcement learning and error detection/correction (Holroyd, Yeung, Coles, \& Cohen, 2005). This theory is supported by animal work, in which it has been demonstrated that midbrain dopamine neurons project to, and can modulate activity in, pyramidal cells in the cingulate cortex (Onn \& Wang, 2005; Williams \& Goldman-Rakic, 1998). Activity in the medial prefrontal cortex can also modulate activity of midbrain dopamine neurons (Carr \& Sesack, 2000; Gao et al., 2007; Gariano \& Groves, 1988; Tong, Overton, \& Clark, 1996), suggesting that signals in the cingulate may also feed back to and modulate the dopamine system.

It is worth noting that the theory that midbrain dopamine neurons signal reward prediction errors has been challenged (Berridge, 2007; Pennartz, 1995; Redgrave \& Gurney, 2006; Redgrave, Prescott, \& Gurney, 1999). Among the challenges are that the phasic dopamine response may be too homogeneous and rapid to signal prediction errors in a range of situations outside those studied in the laboratory (e.g., if it takes time to extract the rewarding properties of a stimulus), that genetically engineered rats that lack dopamine can still learn to seek food rewards (Zhou \& Palmiter, 1995), and that dopamine activity increases following aversive and salient events (Ungless, 2004). Therefore, although the phasic dopamine response is clearly linked to rewards and reward-related behaviors, it may additionally convey messages other than reward prediction error signals, and it may not be necessary for some forms of learning to take place. Nonetheless, this theory has spawned a cornucopia of research, spanning multiple species and methodologies, and it continues to shed light on the neural underpinnings of reward and reinforcement learning. 
Neural correlates of decision option weights. The other important component of reinforcement-learning models is the weight, which represents specific actions, decisions, or stimuli (Barto, 1995; Braver \& Brown, 2003; Egelman et al., 1998). In situations in which the decision options can be mapped onto specific actions (e.g., respond with the left or the right hand, look to one of several possible locations on a screen), weights from computational models may correspond to activity in brain regions responsible for those specific actions (M. X Cohen \& Ranganath, 2007). Although the neural correlates of weights have received less attention than have the neural correlates of prediction errors, a few recent studies have begun to investigate this issue directly (M. X Cohen \& Ranganath, 2007; Haruno \& Kawato, 2006). Indirect evidence from animal studies has provided additional support for the idea that weights for decision options may have a neural correlate. These studies have shown that activity in brain regions involved in representing, planning, and executing motor actions is influenced by the amount or probability of reward that can be obtained for that action (Gold \& Shadlen, 2000; Platt \& Glimcher, 1999; Samejima, Ueda, Doya, \& Kimura, 2005; Schall, 1995, 2005). Furthermore, reward-related activity modulations in motor regions can bias decision making and action selection processes (Gold \& Shadlen, 2000; Schall, 1995, 2005; Sugrue, Corrado, \& Newsome, 2004). Although these findings are not typically discussed in terms of weights from computational learning models, the observations are consistent with the idea that weights in models correspond to activity in sensorimotor systems. This correspondence between weights and activity in neural motor circuits might hold only for situations in which decision options can be mapped onto particular responses.

Neural correlates of learning rates. Learning rates, the parameters that scale the effect of the prediction error on the adjustment of weights, have been shown to correlate with activity in specific brain regions. For example, Tanaka et al. (2004) demonstrated that activity in distinct subregions of the striatum and medial frontal cortex correlates with distinct learning rates, suggesting that learning occurs at different speeds in different brain structures. Behrens, Woolrich, Walton, and Rushworth (2007) demonstrated that intersubject variability in the anterior cingulate BOLD response to reward feedback predicted intersubject variability in learning rates estimated by a computational model. Other fMRI and single-unit recording studies have demonstrated that brain activity in fronto-striatal circuits is predicted by model-estimated learning rates (Barraclough, Conroy, \& Lee, 2004; M. X Cohen \& Ranganath, 2005). Tonic dopamine may be related to learning rate and response vigor (Niv, Daw, Joel, \& Dayan, 2007), although the link between tonic dopamine and learning rate is likely to be complex, because tonic dopamine increases behavioral activity in rats (Evenden \& Robbins, 1983; Ljungberg \& Enquist, 1987) but also attenuates the efficacy of prefrontal cortical inputs into the striatum (Cepeda \& Levine, 1998; Goto \& Grace, 2005), which impairs learning in some tasks (Goto \& Grace, 2005).
The studies reviewed in this section demonstrate that computational reinforcement-learning models, although obviously more simplistic than the processes engaged by the human brain, nonetheless successfully capture several behavioral and neural processes engaged in during learning and decision-making tasks. Although differences and, sometimes, discrepancies between studies exist, together this body of research demonstrates the feasibility and appropriateness of using abstract mathematical models to guide and inform empirical research on the neural bases of decision making. One important caveat here, as with all studies that utilize computational models, is that although equations or outputs of a set of mathematical expressions can explain variance in behavior and in neural activity, one cannot assume that the brain engages these mechanisms or makes these computations intrinsically; rather, one can interpret the findings only to mean that the brain's computations can be more or less effectively estimated through these mathematical expressions.

\section{Empirical Tests of How Reinforcements Might Guide Decision Making}

Reinforcement-learning models provide a framework within which to generate specific hypotheses about how reinforcements may be used to guide future decisions. Some of these predictions are explicitly built into reinforcement-learning models, such as how reinforcements may be used to adjust future decisions (via prediction errors adjusting decision option weights), and other predictions are extensions of these models - for example, accounting for individual differences in learning speed. In this section, I will review recent studies that have used reinforcement-learning models to understand the relationship between how reinforcements are processed and how subsequent decision making is adapted.

Reinforcements guide future decisions. As was stated earlier, the intuitive purpose of devoting brain resources to identifying and evaluating reinforcements is to use that information to adapt future reward-seeking decision making. Although this has thus far received relatively little attention in neuroscience research, one neurocognitive mechanism by which this may occur can be derived from computational reinforcement-learning models, because, in reinforcement-learning models, reinforcements from one trial directly affect the weight on the following trial. In particular, larger prediction errors on one trial will lead to a larger change in weight on the following trial. This is described by the end of Thorndike's (1911) quote, above: "The greater the satisfaction or discomfort, the greater the strengthening or weakening of the bond." This is intrinsic to how the weights are updated: $w_{t}=d+$ $w_{t-1}$. The larger $d$ is, the more the weight will be increased or decreased, depending on whether it was a positive or a negative prediction error. In turn, a larger change in one weight than in the other will increase the bias toward the decision option with the larger weight. Thus, the model makes the simple prediction that larger prediction errors should be associated with an increased likelihood of adjusting behavior and should be accompanied by relatively 
enhanced neural responses in regions thought to be sensitive to prediction errors.

In a recent ERP study (M. X Cohen \& Ranganath, 2007), we tested the prediction that the magnitude of prediction errors should be associated with adjustments in subsequent decisions. We recorded ERPs while subjects played a strategic economic game against a computer opponent. In the game, the subjects had to choose the one of two targets that the computer opponent did not choose, and won or lost on each trial accordingly. This design allowed us to evaluate how neural responses to outcomes related to subsequent decision making. We reasoned that if prediction errors signal the need to adjust future behavior, the FRN should predict adjustments in decision making on the subsequent trial. To test this hypothesis, we separately averaged ERPs during wins and losses according to the decision that was made in the following trial. We found that ERPs following losses were significantly more negative on trials when the subjects chose the opposite target on the following trial, as compared with ERPs during losses when the subjects chose the same target on the following trial (M. X Cohen \& Ranganath, 2007). In other words, loss/opposite trials elicited a larger FRN than did loss/same trials. A similar pattern was observed following wins, although it was not statistically significant. This finding is consistent with the idea that the FRN reflects a neural computation of a prediction error and that prediction errors are used to signal the need to adjust future decision making. To test more explicitly whether the FRN effect reflected a neural prediction error, we examined the responses of a computational reinforcement-learning model, which was based on the equations described earlier, that played the same game against the same computer opponent. Behaviorally, the model performed comparably to how the humans had performed. Consistent with the human ERPs, the model's prediction errors were larger during loss/opposite trials than during loss/same trials. These parallels between the model's prediction errors and human ERPs to decision outcomes support the idea that the FRN effect reflects the computation of negative prediction errors and that it signals the need to adjust behavior. These findings provide a computational mechanism for some previous studies that have shown (through experimental design, not computational modeling) that feedback-related responses in the ventral and orbitofrontal cortices predict adjustments in decision making on subsequent trials (Cools, Clark, Owen, \& Robbins, 2002; O'Doherty, Critchley, Deichmann, \& Dolan, 2003).

How might this prediction error-like signal be used to guide future decisions? In the model, prediction errors are used to strengthen or weaken the weights of the two decision options. When the model loses, the weight for the chosen decision option is weakened relative to that of the nonchosen decision option, and when the model wins, the weight of the chosen decision option is strengthened relative to the weight of the nonchosen decision option. On the basis of the hypothesis presented earlier, that weights in the brain might correspond to the activity of motorrelated systems, we hypothesized that in this task, lateral scalp sites over the motor cortex should exhibit sensitivity to loss versus win feedback when that motor cortex was used to indicate the decision. We found that FRN-like responses over motor cortex electrode sites were lateralized according to the response hand that was used to make the decision. Specifically, a larger FRN was observed over right motor cortex electrodes during feedback following decisions made with the left hand, and a larger FRN was observed over left motor cortex electrodes during feedback following decisions made with the right hand (M. X Cohen \& Ranganath, 2007). This finding suggests that feedback information is processed in motor cortical regions, so that representations of winning responses are strengthened, whereas representations of losing responses are weakened. More generally, these findings add to a growing literature demonstrating that relatively simple reinforcement-learning models can account for behavioral and ERP responses to feedback (Holroyd \& Coles, 2002, 2008; Nieuwenhuis et al., 2002).

These trial-to-trial adjustments in decision making according to feedback may be influenced by genetic factors related to functioning of the dopamine system. Recently, Frank, Moustafa, Haughey, Curran, and Hutchison (2007) demonstrated that a polymorphism on the COMT gene, which is related to dopamine uptake in the prefrontal cortex and striatum (Bilder, Volavka, Lachman, \& Grace, 2004), predicted how likely subjects were to switch their behavior following negative feedback. This work directly implicates the prefrontal dopamine system in trial-to-trial reinforcement-guided adjustments in behavior, consistent with models implicating dopamine in prefrontal-mediated working memory processes (Braver et al., 2001; J. D. Cohen \& Servan-Schreiber, 1993).

A neurocognitive link between feedback on one trial and performance on the following trial is not limited to cases of reward seeking or reward learning. For example, several studies have demonstrated that ERN amplitude during speeded reaction time tasks predicts changes in reaction time on the subsequent trial (Debener et al., 2005; Garavan, Ross, Murphy, Roche, \& Stein, 2002; Gehring, Goss, Coles, Meyer, \& Donchin, 1993; Ridderinkhof, Nieuwenhuis, \& Bashore, 2003). In these cases, internal and/or external indicators of performance may be used to signal the need to adjust future behavior (Fiehler, Ullsperger, \& von Cramon, 2004; Holroyd et al., 2004; Muller, Moller, Rodriguez-Fornells, \& Munte, 2005). These performance indicators might be signaled as prediction errors (Holroyd \& Coles, 2002).

Individual differences and future decisions. The predictions and results outlined in the previous section are normative, in that they prescribe how all individuals should behave. However, human decision making can be variable, and different individuals may respond differently to the same reinforcement. Consider Persons A and B at a roulette table in a casino, each betting $\$ 10$ on black. The ball lands on a black number, leaving both individuals with a total of $\$ 20$. Person A leaves the $\$ 20$ on black to gamble again, but Person B takes the $\$ 20$ off the table and walks away. In this situation, two different individuals made the same decision and received the same reinforcement but used that reinforcement in opposite ways 
to guide their future decision making. Normative theories for how individuals should act cannot account for these individual differences. Most studies using computational reinforcement-learning models are based on such normative assumptions, and learning parameters are typically selected a priori and fixed across subjects (e.g., O'Doherty, Dayan, et al., 2003; Seymour et al., 2004). These models have provided powerful insights into the neural computations of a prediction error, although they traditionally are tested either in passive learning or in simple choice tasks in which there is a "best" or correct response. However, fixing these parameters to be constant across all subjects might not be appropriate in more complex situations, such as those that involve decision making under uncertainty or risk, in which different individuals might interpret the same reinforcement in different ways, as described in the gambling illustration. In other words, models with fixed learning parameters may not capture important intersubject variability, because fixed learning parameters assume that all subjects interpret and use reinforcements in the same way to update weights of decision options. Can reinforcement-learning models be adjusted to account for these individual differences, and can this be used to shed light on the neural processes that support adjusting behavior according to reinforcements?

To examine this, we had subjects choose either relatively high- or low-risk gambles in an fMRI experiment (M. X Cohen \& Ranganath, 2005). Immediately after choosing one of the gambles, the subjects received or did not receive a monetary reward, according to reinforcement contingencies associated with each gamble. The subjects were trained on these contingencies prior to the start of the experiment, which allowed us to focus on how individuals use reinforcements to guide decision making, rather than on trial-and-error learning. In the first step of our analyses, we derived update parameters that were specific to each subject for the different possible reinforcements (winning or not winning high- or low-risk gambles). These parameters were estimated by fitting the model to the subjects' behavioral choices and reinforcements, using a nonlinear estimation procedure (M. X Cohen \& Ranganath, 2005). In the equations presented above, the update parameter is the $l$ in $w_{t+1}=a * l+w_{t}$. To make this more concrete, think back to the roulette example presented earlier, in which $w$ represents the weight of betting on black. The update parameter for winning the roulette gamble, $l$, is positive for Person A but negative for Person B. Thus, on trial $t+1$, the weight of the decision (i.e., whether to bet again) increases for Person A but decreases for Person B. One can imagine a Person $\mathrm{C}$ with $l=0$; his decision to gamble on the next round is unaffected by the outcome on the current round.

Consistent with this example, we found that these update parameters were positive for some subjects and negative for other subjects. These parameters predicted behavioral performance, in terms of the probabilities of choosing particular gambles after winning or losing other gambles. This is perhaps not surprising, considering that the parameters were estimated on the basis of the behavioral data, but this does demonstrate that the model can be used to explain variance in trial-to-trial decisionmaking adjustments, even though the subjects were not learning optimal response patterns, as is typical in reinforcement-learning experiments. The next and more important step was to examine whether these parameters would explain neural activity - and individual differences in neural activity - that might underlie these trial-to-trial adjustments. To do this, we extracted from the fMRI data reinforcement-related activity, separated according to the decision the subjects made on the subsequent trial, and correlated individual differences in the update parameters with individual differences in the fMRI data at each voxel. In this analysis, significant correlations (i.e., activations) indicate regions in which the mathematically estimated update parameters explain how reinforcement-related brain activity predicted the type of gamble the subjects made on the following trial. We found significant correlations in several regions involved in reinforcement processing, including the striatum, amygdala, orbitofrontal cortex, and cingulate cortex. Interestingly, the direction of the effect (i.e., whether increased brain activity in these regions predicted future high- or low-risk decisions) was different in different subjects, which was explained by the update parameters. Had we ignored individual differences (i.e., a normative approach), we would have concluded that reinforcement-related brain activity does not predict future decisions; incorporating individual differences proved critical to elucidating the relation between reinforcementrelated brain activity and adjustments made in future decisions. These findings also demonstrate that the brain engages a reinforcement-learning-like mechanism to adapt ongoing decision making according to outcomes of recent decisions, even during complex decision-making tasks in which there are no correct or best responses.

Other studies have demonstrated that individual differences in the magnitude of neural responses to feedback predict overall learning strategies. Recently, Behrens et al. (2007) reported a similar finding: that intersubject variability in learning rates estimated by a computational model correlated with the BOLD response in the anterior cingulate cortex during reward feedback. Also, Wrase et al. (2007) demonstrated that individual differences in the BOLD response to reward and punishment feedback predicted how subjects adapted their reaction times on subsequent trials. Researchers have also used ERP to link individual differences in neural responses to feedback to individual differences in decision making and reward learning. With regard to the ERN, Hewig et al. (2007) demonstrated that subjects who were more likely to choose high-risk options in a blackjack game had smaller ERN amplitudes when taking another card (gamble), as compared with subjects who played more cautiously. Frank et al. (2005) demonstrated a similar finding, showing that subjects who learned more by seeking positive outcomes had smaller ERNs, as compared with subjects who learned more by avoiding negative outcomes. Assuming that the ERN reflects a prediction error (Holroyd \& Coles, 2002), one possibility is that individuals who are less sensitive to negative feedback (which may produce a smaller ERN) are more likely to seek out positive outcomes, even at a 
potentially higher risk. Consistent with this possibility, substance abusers and highly impulsive individuals have a smaller ERN than do controls and less impulsive subjects (Franken, van Strien, Franzek, \& van de Wetering, 2007; Potts, George, Martin, \& Barratt, 2006). With regard to the FRN, Yeung and Sanfey (2004) showed that subjects who were more likely to engage in risk-seeking decisions after losing money in a gamble had a greater FRN. These individual-difference studies also suggest that the ERN/ FRN reflects trait differences in learning style or other stable personality factors.

Individual differences and reward prediction errors. The analyses described in the previous section address individual differences in update parameters in reinforcement-learning models that are assumed to be known a priori. In a further step, we wished to examine whether individual differences would also be relevant for models with reward prediction errors - that is, when the outcomes of decisions are not assumed to be known a priori but can be learned and can change throughout the course of the experiment. In particular, we focused on the learning rates that describe how the prediction error adjusts the weights. As was mentioned earlier, a large learning rate means that the prediction error strongly influences the adjustment of the weight, whereas a small learning rate (e.g., close to 0) means that the prediction error influences the weights only slightly. Learning rates typically are selected a priori and fixed across subjects (e.g., O'Doherty, Dayan, et al., 2003; Seymour et al., 2004).

We directly tested how models that incorporate individually estimated learning parameters compare with models that use fixed learning parameters. To do this, we (M. X Cohen, 2007) reanalyzed data from an fMRI study in which subjects chose between a high- and a low-risk gamble. In the model for this set of analyses, we included a prediction error, so that the model comparison was similar to other models used in previous studies. Testing for neural correlates of a prediction error in fMRI data involves having a model estimate a prediction error on each trial for each subject and using that estimate as a regression term; voxels in which activity correlates with the prediction error specified by the model will have significant activations. We compared three different models: (1) a model in which learning parameters were estimated for each subject individually; (2) a model in which learning parameters were estimated for each subject and were then averaged together across all subjects to create a group estimate of the learning parameters; and (3) a model that used fixed learning parameters across all subjects, taken from published studies.

Overall, the model's outputs predicted subjects' trialto-trial choice strategies and neural activity in several limbic and prefrontal regions, including the midbrain, ventral striatum, cingulate cortex, and orbitofrontal cortex. These activations were observed both when the individual-difference and when the group models were used; relatively few activations were observed when the fixed model was used. We directly statistically compared the amount of variance explained by the different models at each voxel and found that the group and individual parameter models explained more variance in the fMRI time courses in several regions, including the ventral and dorsal striatum, cingulate cortex, and orbitofrontal and dorsolateral prefrontal cortex. Importantly, the direction of the effect (whether the BOLD response was positively or negatively correlated with the model predictions) differed across subjects. This variance was captured by the individual-difference models, but not by the fixed model. The differences in model fit were not likely due to the differences in the number of model parameters, because the fits were not globally and generally better and, in some cases, the most highly parameterized models did not fit the data as well as the model with fewer parameters. However, even if the number of parameters had contributed to some of the results, given that the direction of the effects reversed for different subjects and this was captured by the better-fitting model, it still demonstrates that simple models with a fixed learning rate across subjects might not be appropriate in some cases. Finally, we fit the model to the behavioral data and found that how well the model fit the behavioral data predicted how well the model fit the fMRI data. In other words, in subjects for whom trial-totrial adjustments in decision making were relatively better fit by the reinforcement-learning model, brain activity was relatively better characterized by the reinforcementlearning model.

As in the analyses presented in the previous section, individual differences in estimated reinforcement-learning parameters proved critical for characterizing these processes, because models that incorporated individual learning parameters explained significantly more variance in the fMRI data than did a model using fixed learning parameters. This does not mean that choosing learning parameters a priori to be the same for all subjects is incorrect or inappropriate. Fixed parameters might be appropriate in passive-learning experiments or in simple decisionmaking situations in which the optimal response is always to maintain rewarded behaviors and avoid punished behaviors. However, in more complex situations in which different individuals evaluate and utilize reinforcements in different ways, models with a priori chosen parameters may not adequately characterize reinforcement-learning processes. These findings are also in accordance with the results of previous studies that have demonstrated that individual differences in reward prediction errorlike responses correlate with stable individual-difference markers, including personality and genotype (Abler et al., 2006; M. X Cohen, Young, Baek, Kessler, \& Ranganath, 2005; Yacubian et al., 2007). In a similar vein, Brown and Braver (2007) recently demonstrated that individual differences in gambling tendencies predicted crossover interactions in performance and anterior cingulate activity related to response conflict and error likelihood. These differences were accounted for by a computational model of performance-monitoring functions of the anterior cingulate (Brown \& Braver, 2008).

\section{Conclusions and Future Directions}

Here, I have provided an overview of computational reinforcement-learning models, how they have been linked 
to psychological and neurobiological processes, and how they can be used to understand the neurocomputational mechanisms by which reinforcements might guide decision making. Although this review was not selective to the medial frontal cortex, many findings discussed here involved this region, which speaks to the critical involvement of the medial frontal cortex in reinforcement-guided decision making (Rushworth, Buckley, Behrens, Walton, \& Bannerman, 2007; Rushworth, Walton, Kennerley, \& Bannerman, 2004). This review also has focused on empirical studies that have examined how reinforcement signals might be used to adapt future behavior and on the relatively simple reinforcement-learning models that have been used to capture these neural dynamics. This represents one small but important component of flexible goal-directed behavior; other cognitive operations that are critical to reinforcement learning include working memory (Braver et al., 2001; Dehaene \& Changeux, 2000; Floresco \& Magyar, 2006), attention (Dehaene \& Changeux, 2000; Lee, Youn, O, Gallagher, \& Holland, 2006; Maddox, Bohil, \& Dodd, 2003), inhibition of impulsive reward-seeking tendencies (Frank, 2006; Kalenscher, Ohmann, \& Güntürkün, 2006; King, Tenney, Rossi, Colamussi, \& Burdick, 2003), reward processing in the orbitofrontal cortex (Kringelbach, 2005; Kringelbach \& Rolls, 2004), and action selection in the anterior cingulate cortex (Holroyd \& Coles, 2008; Rushworth et al., 2007; Rushworth et al., 2004).

Adjusting reward-seeking behavior according to reinforcements is a fundamental function of the brain; the studies reviewed in the latter sections of this review represent part of a nascent effort to bring previous research forward one step by focusing less on how reinforcements are processed per se and more on how reinforcements may be used to guide future decision making. Theoretical and empirical advances will further elucidate our understanding of these processes. One avenue likely to provide fruitful insights is by developing increasingly sophisticated models that can account for and make predictions regarding reinforcementguided decision adjustments. Recently, more sophisticated abstract models have been put forth and have been supported with empirical data from fMRI (Daw et al., 2006; Hampton et al., 2006). These models have shed insight into mathematically characterizable brain processes, and in some cases, they outperform simple reinforcement-learning models (Hampton et al., 2006). However, these models reflect abstract cognitive processes; they are not specifically rooted in neurobiology, nor do they make specific predictions regarding the functions or activities of different brain regions. Models that are constrained by neurobiology may help account for and make predictions regarding the specific functions of different brain regions (Frank \& Claus, 2006). A related point is that neurochemical systems such as dopamine are known to influence learning and rewardseeking behavior (Blum et al., 2000; Schultz, 1998; Spanagel \& Weiss, 1999) and reward-related activity in limbic and frontal brain regions (Everitt et al., 1999; Koob, 1999). In more biologically constrained models, researcher are beginning to take into consideration the specific roles that dopamine might have during reinforcement learning (Frank,
2005; Niv et al., 2007). Future research could test specific hypotheses regarding the role of dopamine agents and genetic polymorphisms on behavioral and neural correlates of reinforcement-guided decision adjustments (e.g., Frank et al., 2007).

However, modeling is not critical or necessary for elucidating neural mechanisms of how reinforcements are used to guide future decisions. Several research groups have demonstrated, without using computational modeling, that reinforcement-related activity in regions including the orbitofrontal cortex and striatum predict decisions made on subsequent trials (Cools et al., 2002; Cools, Lewis, Clark, Barker, \& Robbins, 2007; O'Doherty, Critchley, et al. 2003; Wrase et al., 2007). Other studies have revealed neural correlates of reward prediction errors through experimental design instead of computational modeling (e.g., Abler et al., 2006; McClure et al., 2003; Rolls et al., 2008). Indeed, modeling could be disadvantageous if it narrows researchers' theoretical approaches, constrains data analysis, or precludes interpretations of findings not predicted by the model. The advantages of incorporating computational modeling into empirical research include the ability to generate and test novel hypotheses and the ability to compare findings from multiple species, methodologies, and brain regions, using a common and mathematically grounded framework.

\section{AUTHOR NOTE}

I thank Charan Ranganath for his help and support in the research detailed here, anonymous reviewers for their useful comments and suggestions, and the National Institute on Drug Abuse for providing support through an NRSA. Correspondence concerning this article should be addressed to M. X Cohen, who is now at the Department of Psychology, University of Arizona, 1503 E. University Blvd., Tucson, AZ 85721 (e-mail: mikexcohen@gmail.com).

\section{REFERENCES}

Abler, B., Walter, H., Erk, S., Kammerer, H., \& Spitzer, M. (2006). Prediction error as a linear function of reward probability is coded in human nucleus accumbens. Neurolmage, 31, 790-795.

Aron, A. R., Shohamy, D., Clark, J., Myers, C., Gluck, M. A., \& POLDRACK, R. A. (2004). Human midbrain sensitivity to cognitive feedback and uncertainty during classification learning. Journal of Neurophysiology, 92, 1144-1152.

Aston-Jones, G., \& Cohen, J. D. (2005). Adaptive gain and the role of the locus coeruleus-norepinephrine system in optimal performance. Journal of Comparative Neurology, 493, 99-110.

Barraclough, D. J., Conroy, M. L., \& Lee, D. (2004). Prefrontal cortex and decision making in a mixed-strategy game. Nature Neuroscience, 7, 404-410.

Barto, A. G. (1995). Reinforcement learning. In M. A. Arbib (Ed.), Handbook of brain theory and neural networks (pp. 804-809). Cambridge, MA: MIT Press.

BAyer, H. M., \& Glimcher, P. W. (2005). Midbrain dopamine neurons encode a quantitative reward prediction error signal. Neuron, 47, 129-141.

Bayer, H. M., LAU, B., \& Glimcher, P. W. (2007). Statistics of midbrain dopamine neuron spike trains in the awake primate. Journal of Neurophysiology, 98, 1428-1439.

Behrens, T. E., Woolrich, M. W., Walton, M. E., \& Rushworth, M. F. (2007). Learning the value of information in an uncertain world. Nature Neuroscience, 10, 1214-1221.

BERRIDGE, K. C. (2007). The debate over dopamine's role in reward: The case for incentive salience. Psychopharmacology, 191, 391-431.

Bilder, R. M., Volavka, J., Lachman, H. M., \& Grace, A. A. (2004). 
The catechol-O-methyltransferase polymorphism: Relations to the tonic-phasic dopamine hypothesis and neuropsychiatric phenotypes. Neuropsychopharmacology, 29, 1943-1961.

Blum, K., Braverman, E. R., Holder, J. M., Lubar, J. F., Monastra, V. J., Miller, D., ET AL. (2000). Reward deficiency syndrome: A biogenetic model for the diagnosis and treatment of impulsive, addictive, and compulsive behaviors. Journal of Psychoactive Drugs, 32 (Suppl., i-iv), 1-112.

Bogacz, R., Brown, E., Moehlis, J., Holmes, P., \& Cohen, J. D. (2006). The physics of optimal decision making: A formal analysis of models of performance in two-alternative forced-choice tasks. Psychological Review, 113, 700-765.

Bogacz, R., McClure, S. M., Li, J., Cohen, J. D., \& Montague, P. R. (2007). Short-term memory traces for action bias in human reinforcement learning. Brain Research, 1153, 111-121.

Braver, T. S., Barch, D. M., Keys, B. A., Carter, C. S., Cohen, J. D., KAYE, J. A., ET AL. (2001). Context processing in older adults: Evidence for a theory relating cognitive control to neurobiology in healthy aging. Journal of Experimental Psychology: General, 130, 746-763.

Braver, T. S., \& Brown, J. W. (2003). Principles of pleasure prediction: Specifying the neural dynamics of human reward learning. Neuron, 38, 150-152.

Brown, J. W., \& Braver, T. S. (2007). Risk prediction and aversion by anterior cingulate cortex. Cognitive, Affective, \& Behavioral Neuroscience, 7, 266-277.

Brown, J. W., \& Braver, T. S. (2008). A computational model of risk, conflict, and individual difference effects in the anterior cingulate cortex. Brain Research, 1202, 99-108.

CAmerer, C. F. (2003). Behavioral game theory: Experiments in strategic interaction. Princeton: Princeton University Press.

CARDINAL, R. N. (2006). Neural systems implicated in delayed and probabilistic reinforcement. Neural Networks, 19, 1277-1301.

Carr, D. B., \& SeSack, S. R. (2000). Projections from the rat prefrontal cortex to the ventral tegmental area: Target specificity in the synaptic associations with mesoaccumbens and mesocortical neurons. Journal of Neuroscience, 20, 3864-3873.

Cepeda, C., \& Levine, M. S. (1998). Dopamine and N-methyl-Daspartate receptor interactions in the neostriatum. Developmental Neuroscience, 20, 1-18.

Cohen, J. D., McClure, S. M., \& Yu, A. J. (2007). Should I stay or should I go? How the human brain manages the trade-off between exploitation and exploration. Philosophical Transactions of the Royal Society B, 362, 933-942.

Cohen, J. D., \& Servan-Schreiber, D. (1993). A theory of dopamine function and its role in cognitive deficits in schizophrenia. Schizophrenia Bulletin, 19, 85-104.

Cohen, M. X (2007). Individual differences and the neural representations of reward expectation and reward prediction error. Social Cognitive \& Affective Neuroscience, 2, 20-30.

Cohen, M. X, Elger, C. E., \& Ranganath, C. (2007). Reward expectation modulates feedback-related negativity and EEG spectra. Neurolmage, 35, 968-978.

Cohen, M. X, \& Ranganath, C. (2005). Behavioral and neural predictors of upcoming decisions. Cognitive, Affective, \& Behavioral Neuroscience, 5, 117-126.

Cohen, M. X, \& Ranganath, C. (2007). Reinforcement learning signals predict future decisions. Journal of Neuroscience, 27, 371-378.

Cohen, M. X, Young, J., Baek, J. M., Kessler, C., \& Ranganath, C. (2005). Individual differences in extraversion and dopamine genetics predict neural reward responses. Cognitive Brain Research, 25, 851-861.

Cools, R., Clark, L., Owen, A. M., \& Robbins, T. W. (2002). Defining the neural mechanisms of probabilistic reversal learning using eventrelated functional magnetic resonance imaging. Journal of Neuroscience, 22, 4563-4567.

Cools, R., Lewis, S. J., Clark, L., Barker, R. A., \& Robbins, T. W. (2007). L-DOPA disrupts activity in the nucleus accumbens during reversal learning in Parkinson's disease. Neuropsychopharmacology, 32, 180-189.

Daw, N. D., \& Doya, K. (2006). The computational neurobiology of learning and reward. Current Opinion in Neurobiology, 16, 199-204.

Daw, N. D., O’Doherty, J. P., Dayan, P., Seymour, B., \& Dolan,
R. J. (2006). Cortical substrates for exploratory decisions in humans. Nature, 441, 876-879.

Dayan, P., \& Balleine, B. W. (2002). Reward, motivation, and reinforcement learning. Neuron, 36, 285-298.

Debener, S., Ullsperger, M., Siegel, M., Fiehler, K., von Cramon, D. Y., \& ENGEL, A. K. (2005). Trial-by-trial coupling of concurrent electroencephalogram and functional magnetic resonance imaging identifies the dynamics of performance monitoring. Journal of Neuroscience, $\mathbf{2 5}$, 11730-11737.

Dehaene, S., \& Changeux, J. P. (2000). Reward-dependent learning in neuronal networks for planning and decision making. Progress in Brain Research, 126, 217-229.

Delgado, M. R., Miller, M. M., Inati, S., \& Phelps, E. A. (2005). An fMRI study of reward-related probability learning. NeuroImage, 24, 862-873.

DitTerich, J. (2006). Stochastic models of decisions about motion direction: Behavior and physiology. Neural Networks, 19, 981-1012.

Egelman, D. M., Person, C., \& Montague, P. R. (1998). A computational role for dopamine delivery in human decision-making. Journal of Cognitive Neuroscience, 10, 623-630.

EVEnden, J. L., \& Robbins, T. W. (1983). Increased response switching, perseveration and perseverative switching following d-amphetamine in the rat. Psychopharmacology, 80, 67-73.

Everitt, B. J., Parkinson, J. A., Olmstead, M. C., Arroyo, M., RobLEDO, P., \& RobBins, T. W. (1999). Associative processes in addiction and reward: The role of amygdala-ventral striatal subsystems. In J. F. McGinty (Ed.), Advancing from the ventral striatum to the extended amygdala: Implications for neuropsychiatry and drug abuse (Annals of the New York Academy of Sciences, Vol. 877, pp. 412-438). New York: New York Academy of Sciences.

Fiehler, K., Ullsperger, M., \& von Cramon, D. Y. (2004). Neural correlates of error detection and error correction: Is there a common neuroanatomical substrate? European Journal of Neuroscience, 19, 3081-3087.

Filoteo, J. V., Maddox, W. T., Simmons, A. N., Ing, A. D., Cagigas, X. E., Matthews, S., \& Paulus, M. P. (2005). Cortical and subcortical brain regions involved in rule-based category learning. NeuroReport, 16, 111-115.

Floresco, S. B., \& Magyar, O. (2006). Mesocortical dopamine modulation of executive functions: Beyond working memory. Psychopharmacology, 188, 567-585.

FranK, M. J. (2005). Dynamic dopamine modulation in the basal ganglia: A neurocomputational account of cognitive deficits in medicated and nonmedicated Parkinsonism. Journal of Cognitive Neuroscience, 17, 51-72.

Frank, M. J. (2006). Hold your horses: A dynamic computational role for the subthalamic nucleus in decision making. Neural Networks, 19, 1120-1136.

Frank, M. J., \& Claus, E. D. (2006). Anatomy of a decision: Striatoorbitofrontal interactions in reinforcement learning, decision making, and reversal. Psychological Review, 113, 300-326.

Frank, M. J., Moustafa, A. A., Haughey, H. M., Curran, T., \& Hutchison, K. E. (2007). Genetic triple dissociation reveals multiple roles for dopamine in reinforcement learning. Proceedings of the $\mathrm{Na}$ tional Academy of Sciences, 104, 16311-16316.

Frank, M. J., Seeberger, L. C., \& O'Reilly, R. C. (2004). By carrot or by stick: Cognitive reinforcement learning in Parkinsonism. Science, 306, 1940-1943.

Frank, M. J., Woroch, B. S., \& Curran, T. (2005). Error-related negativity predicts reinforcement learning and conflict biases. Neuron, 47, 495-501.

Franken, I. H., van Strien, J. W., Franzek, E. J., \& van de WeterING, B. J. (2007). Error-processing deficits in patients with cocaine dependence. Biological Psychology, 75, 45-51.

GaO, M., LiU, C. L., Yang, S., Jin, G. Z., Bunney, B. S., \& ShI, W. X. (2007). Functional coupling between the prefrontal cortex and dopamine neurons in the ventral tegmental area. Journal of Neuroscience, 27, 5414-5421.

Garavan, H., Ross, T. J., Murphy, K., Roche, R. A., \& Stein, E. A. (2002). Dissociable executive functions in the dynamic control of behavior: Inhibition, error detection, and correction. NeuroImage, 17, 1820-1829.

Gariano, R. F., \& Groves, P. M. (1988). Burst firing induced in mid- 
brain dopamine neurons by stimulation of the medial prefrontal and anterior cingulate cortices. Brain Research, 462, 194-198.

Gehring, W. J., Goss, B., Coles, M. G., Meyer, D. E., \& Donchin, E. (1993). A neural system for error detection and compensation. Psychological Science, 4, 385-390.

Glimcher, P. W., Dorris, M. C., \& Bayer, H. M. (2005). Physiological utility theory and the neuroeconomics of choice. Games \& Economic Behavior, 52, 213-256.

Gold, J. I., \& Shadlen, M. N. (2000). Representation of a perceptual decision in developing oculomotor commands. Nature, 404, 390-394.

Gold, J. I., \& Shadlen, M. N. (2002). Banburismus and the brain: Decoding the relationship between sensory stimuli, decisions, and reward. Neuron, 36, 299-308.

Goto, Y., \& Grace, A. A. (2005). Dopaminergic modulation of limbic and cortical drive of nucleus accumbens in goal-directed behavior. Nature Neuroscience, $\mathbf{8}, 805-812$.

Hajcak, G., Holroyd, C. B., Moser, J. S., \& Simons, R. F. (2005). Brain potentials associated with expected and unexpected good and bad outcomes. Psychophysiology, 42, 161-170.

Hajcak, G., Moser, J. S., Holroyd, C. B., \& Simons, R. F. (2007). It's worse than you thought: The feedback negativity and violations of reward prediction in gambling tasks. Psychophysiology, 44, 905-912.

Hampton, A. N., Bossaerts, P., \& O’Doherty, J. P. (2006). The role of the ventromedial prefrontal cortex in abstract state-based inference during decision making in humans. Journal of Neuroscience, 26, 8360-8367.

Haruno, M., \& Kawato, M. (2006). Different neural correlates of reward expectation and reward expectation error in the putamen and caudate nucleus during stimulus-action-reward association learning. Journal of Neurophysiology, 95, 948-959.

Haruno, M., Kuroda, T., Doya, K., Toyama, K., Kimura, M., SameJIMA, K., ET AL. (2004). A neural correlate of reward-based behavioral learning in caudate nucleus: A functional magnetic resonance imaging study of a stochastic decision task. Journal of Neuroscience, $\mathbf{2 4}$, 1660-1665.

Hewig, J., Trippe, R., Hecht, H., Coles, M. G., Holroyd, C. B., \& Miltner, W. H. (2007). Decision-making in blackjack: An electrophysiological analysis. Cerebral Cortex, 17, 865-877.

Hollerman, J. R., \& Schultz, W. (1998). Dopamine neurons report an error in the temporal prediction of reward during learning. Nature Neuroscience, 1, 304-309.

Holroyd, C. B., \& Coles, M. G. (2002). The neural basis of human error processing: Reinforcement learning, dopamine, and the errorrelated negativity. Psychological Review, 109, 679-709.

Holroyd, C. B., \& Coles, M. G. (2008). Dorsal anterior cingulate integrates reinforcement history to guide voluntary behavior. Cortex, 44, 548-559.

Holroyd, C. B., Nieuwenhuis, S., Yeung, N., \& Cohen, J. D. (2003). Errors in reward prediction are reflected in the event-related brain potential. NeuroReport, 14, 2481-2484.

Holroyd, C. B., Nieuwenhuis, S., Yeung, N., Nystrom, L., Mars, R. B., Coles, M. G., \& Cohen, J. D. (2004). Dorsal anterior cingulate cortex shows fMRI response to internal and external error signals. Nature Neuroscience, 7, 497-498.

Holroyd, C. B., Yeung, N., Coles, M. G., \& Cohen, J. D. (2005). A mechanism for error detection in speeded response time tasks. Journal of Experimental Psychology: General, 134, 163-191.

Houk, J. C., \& Wise, S. P. (1995). Distributed modular architectures linking basal ganglia, cerebellum, and cerebral cortex: Their role in planning and controlling action. Cerebral Cortex, 5, 95-110.

JoEL, D., Niv, Y., \& RuPPIN, E. (2002). Actor-critic models of the basal ganglia: New anatomical and computational perspectives. Neural Networks, 15, 535-547.

Kalenscher, T., Ohmann, T., \& Güntürkün, O. (2006). The neuroscience of impulsive and self-controlled decisions. International Journal of Psychophysiology, 62, 203-211.

King, J. A., Tenney, J., Rossi, V., Colamussi, L., \& Burdick, S. (2003). Neural substrates underlying impulsivity. In J. A. King, C. F. Ferris, \& I. I. Lederhendler (Eds.), Roots of mental illness in children (Annals of the New York Academy of Sciences, Vol. 1008, pp. 160-169). New York: New York Academy of Sciences.

Knutson, B., \& Wimmer, G. E. (2007). Splitting the difference: How does the brain code reward episodes? In B. W. Balleine, K. Doya, J. O'Doherty, \& M. Sakagumi (Eds.), Reward and decision making in corticobasal ganglia networks (Annals of the New York Academy of Sciences, Vol. 1104, pp. 54-69). New York: New York Academy of Sciences.

Коов, G. F. (1999). The role of the striatopallidal and extended amygdala systems in drug addiction. In J. F. McGinty (Ed.), Advancing from the ventral striatum to the extended amygdala: Implications for neuropsychiatry and drug abuse (Annals of the New York Academy of Sciences, Vol. 877, pp. 445-460). New York: New York Academy of Sciences.

KrawcZyK, D. C. (2002). Contributions of the prefrontal cortex to the neural basis of human decision making. Neuroscience \& Biobehavioral Reviews, 26, 631-664.

KRINGELBACH, M. L. (2005). The human orbitofrontal cortex: Linking reward to hedonic experience. Nature Reviews Neuroscience, 6 , 691-702.

KringelbaCh, M. L., \& Rolls, E. T. (2004). The functional neuroanatomy of the human orbitofrontal cortex: Evidence from neuroimaging and neuropsychology. Progress in Neurobiology, 72, 341-372.

Lee, H. J., Youn, J. M., O, M. J., Gallagher, M., \& Holland, P. C. (2006). Role of substantia nigra-amygdala connections in surpriseinduced enhancement of attention. Journal of Neuroscience, 26, 6077-6081.

LJungBerg, T., \& ENQuist, M. (1987). Disruptive effects of low doses of d-amphetamine on the ability of rats to organize behaviour into functional sequences. Psychopharmacology, 93, 146-151.

Maddox, W. T., BohIL, C. J., \& DodD, J. L. (2003). Linear transformations of the payoff matrix and decision criterion learning in perceptual categorization. Journal of Experimental Psychology: Learning, Memory, \& Cognition, 29, 1174-1193.

McClure, S. M., Berns, G. S., \& Montague, P. R. (2003). Temporal prediction errors in a passive learning task activate human striatum. Neuron, 38, 339-346.

Montague, P. R., \& Berns, G. S. (2002). Neural economics and the biological substrates of valuation. Neuron, 36, 265-284.

Montague, P. R., Dayan, P., \& Sejnowski, T. J. (1996). A framework for mesencephalic dopamine systems based on predictive Hebbian learning. Journal of Neuroscience, 16, 1936-1947.

Montague, P. R., Hyman, S. E., \& Cohen, J. D. (2004). Computational roles for dopamine in behavioural control. Nature, 431, 760-767.

Muller, S. V., Moller, J., Rodriguez-Fornells, A., \& Munte, T. F. (2005). Brain potentials related to self-generated and external information used for performance monitoring. Clinical Neurophysiology, 116, 63-74.

Murray, G. K., Corlett, P. R., Clark, L., Pessiglione, M., BlackWell, A. D., Honey, G., ET AL. (2008). Substantia nigra/ventral tegmental reward prediction error disruption in psychosis. Molecular Psychiatry, 13, 267-276.

NaKahara, H., Itoh, H., Kawagoe, R., Takikawa, Y., \& Hikosaka, O. (2004). Dopamine neurons can represent context-dependent prediction error. Neuron, 41, 269-280.

Nieuwenhuis, S., Holroyd, C. B., Mol, N., \& Coles, M. G. (2004). Reinforcement-related brain potentials from medial frontal cortex: Origins and functional significance. Neuroscience \& Biobehavioral Reviews, 28, 441-448.

Nieuwenhuis, S., Ridderinkhof, K. R., Talsma, D., Coles, M. G., Holroyd, C. B., KoK, A., \& van der Molen, M. W. (2002). A computational account of altered error processing in older age: Dopamine and the error-related negativity. Cognitive, Affective, \& Behavioral Neuroscience, 2, 19-36.

Niv, Y., DaW, N. D., Joel, D., \& Dayan, P. (2007). Tonic dopamine: Opportunity costs and the control of response vigor. Psychopharmacology, 191, 507-520.

O’Doherty, J. [P.], Critchley, H., Deichmann, R., \& Dolan, R. J. (2003). Dissociating valence of outcome from behavioral control in human orbital and ventral prefrontal cortices. Journal of Neuroscience, 23, 7931-7939.

O’Doherty, J. P., Dayan, P., Friston, K., Critchley, H., \& Dolan, R. J. (2003). Temporal difference models and reward-related learning in the human brain. Neuron, 38, 329-337.

O’Doherty, J. P., Dayan, P., Schultz, J., Deichmann, R., FrisTON, K., \& Dolan, R. J. (2004). Dissociable roles of ventral and dorsal striatum in instrumental conditioning. Science, 304, 452-454. 
O’Doherty, J. P., Hampton, A., \& Kim, H. (2007). Model-based fMRI and its application to reward learning and decision making. In B. W. Balleine, K. Doya, J. O'Doherty, \& M. Sakagumi (Eds.), Reward and decision making in corticobasal ganglia networks (Annals of the New York Academy of Sciences, Vol. 1104, pp. 35-53). New York: New York Academy of Sciences.

ONN, S. P., \& WANG, X. B. (2005). Differential modulation of anterior cingulate cortical activity by afferents from ventral tegmental area and mediodorsal thalamus. European Journal of Neuroscience, 21, 2975-2992.

O'Reilly, R. C. (2006). Biologically based computational models of high-level cognition. Science, 314, 91-94.

Pennartz, C. M. (1995). The ascending neuromodulatory systems in learning by reinforcement: Comparing computational conjectures with experimental findings. Brain Research Reviews, 21, 219-245.

Platt, M. L., \& Glimcher, P. W. (1999). Neural correlates of decision variables in parietal cortex. Nature, 400, 233-238.

Potts, G. F., George, M. R., Martin, L. E., \& Barratt, E. S. (2006). Reduced punishment sensitivity in neural systems of behavior monitoring in impulsive individuals. Neuroscience Letters, 397, 130-134.

Ramnani, N., Elliott, R., Athwal, B. S., \& Passingham, R. E. (2004). Prediction error for free monetary reward in the human prefrontal cortex. Neurolmage, 23, 777-786.

RATCLIFF, R. (2002). A diffusion model account of response time and accuracy in a brightness discrimination task: Fitting real data and failing to fit fake but plausible data. Psychonomic Bulletin \& Review, 9, 278-291.

Redgrave, P., \& Gurney, K. (2006). The short-latency dopamine signal: A role in discovering novel actions? Nature Reviews Neuroscience, 7, 967-975.

Redgrave, P., Prescott, T. J., \& Gurney, K. (1999). Is the shortlatency dopamine response too short to signal reward error? Trends in Neurosciences, 22, 146-151.

RidderinkHof, K. R., Nieuwenhuis, S., \& BASHORE, T. R. (2003). Errors are foreshadowed in brain potentials associated with action monitoring in cingulate cortex in humans. Neuroscience Letters, 348, 1-4.

Rodriguez, P. F., Aron, A. R., \& Poldrack, R. A. (2006). Ventralstriatal/nucleus-accumbens sensitivity to prediction errors during classification learning. Human Brain Mapping, 27, 306-313.

Rolls, E. T., McCabe, C., \& Redoute, J. (2008). Expected value, reward outcome, and temporal difference error representations in a probabilistic decision task. Cerebral Cortex, 18, 652-663.

Ruchsow, M., Grothe, J., Spitzer, M., \& Kiefer, M. (2002). Human anterior cingulate cortex is activated by negative feedback: Evidence from event-related potentials in a guessing task. Neuroscience Letters, 325, 203-206

Rushworth, M. F., Buckley, M. J., Behrens, T. E., Walton, M. E., \& BANNERMAN, D. M. (2007). Functional organization of the medial frontal cortex. Current Opinion in Neurobiology, 17, 220-227.

Rushworth, M. F., Walton, M. E., Kennerley, S. W., \& BannerMAN, D. M. (2004). Action sets and decisions in the medial frontal cortex. Trends in Cognitive Sciences, 8, 410-417.

Samejima, K., Ueda, Y., Doya, K., \& Kimura, M. (2005). Representation of action-specific reward values in the striatum. Science, $\mathbf{3 1 0}$, 1337-1340.

Schall, J. D. (1995). Neural basis of saccade target selection. Reviews in the Neurosciences, 6, 63-85.

Schall, J. D. (2005). Decision making. Current Biology, 15, R9-R11.

Schultz, W. (1998). Predictive reward signal of dopamine neurons. Journal of Neurophysiology, 80, 1-27.

Schultz, W. (2001). Reward signaling by dopamine neurons. Neuroscientist, 7, 293-302.

Schultz, W. (2006). Behavioral theories and the neurophysiology of reward. Annual Review of Psychology, 57, 87-115.

Schultz, W., Dayan, P., \& Montague, P. R. (1997). A neural substrate of prediction and reward. Science, 275, 1593-1599.
Schultz, W., \& Dickinson, A. (2000). Neuronal coding of prediction errors. Annual Review of Neuroscience, 23, 473-500.

Seymour, B., Daw, N., Dayan, P., Singer, T., \& Dolan, R. (2007). Differential encoding of losses and gains in the human striatum. Journal of Neuroscience, 27, 4826-4831.

Seymour, B., O’Doherty, J. P., Dayan, P., Koltzenburg, M., Jones, A. K., Dolan, R. J., ET AL. (2004). Temporal difference models describe higher-order learning in humans. Nature, 429, 664-667.

Simen, P., Cohen, J. D., \& Holmes, P. (2006). Rapid decision threshold modulation by reward rate in a neural network. Neural Networks, $\mathbf{1 9}$, 1013-1026.

SPANAGEL, R., \& WeIss, F. (1999). The dopamine hypothesis of reward: Past and current status. Trends in Neurosciences, 22, 521-527.

Sugrue, L. P., Corrado, G. S., \& Newsome, W. T. (2004). Matching behavior and the representation of value in the parietal cortex. Science, 304, 1782-1787.

SURI, R. E. (2002). TD models of reward predictive responses in dopamine neurons. Neural Networks, 15, 523-533.

SURI, R. E., \& Schultz, W. (1999). A neural network model with dopamine-like reinforcement signal that learns a spatial delayed response task. Neuroscience, 91, 871-890.

Sutton, R. S., \& Barto, A. G. (1990). Time-derivative models of Pavlovian reinforcement. In M. Gabriel \& J. Moore (Eds.), Learning and computational neuroscience: Foundations of adaptive networks (pp. 539-602). Cambridge, MA: MIT Press.

Sutton, R. S., \& Barto, A. G. (1998). Reinforcement learning. Cambridge, MA: MIT Press.

Tanaka, S. C., Doya, K., OKada, G., Ueda, K., Окamoto, Y., \& YaMAWAKI, S. (2004). Prediction of immediate and future rewards differentially recruits cortico-basal ganglia loops. Nature Neuroscience, 7, 887-893.

Thorndike, E. L. (1911). Animal intelligence: Experimental studies. New York: Macmillan.

Tong, Z. Y., Overton, P. G., \& Clark, D. (1996). Stimulation of the prefrontal cortex in the rat induces patterns of activity in midbrain dopaminergic neurons which resemble natural burst events. Synapse, 22, 195-208.

Ungless, M. A. (2004). Dopamine: The salient issue. Trends in Neurosciences, 27, 702-706.

Williams, S. M., \& Goldman-Rakic, P. S. (1998). Widespread origin of the primate mesofrontal dopamine system. Cerebral Cortex, $\mathbf{8}, 321-345$

Wrase, J., Kahnt, T., Schlagenhauf, F., Beck, A., Cohen, M. X, Knutson, B., \& Heinz, A. (2007). Different neural systems adjust motor behavior in response to reward and punishment. NeuroImage, 36, 1253-1262.

Yacubian, J., Glascher, J., Schroeder, K., Sommer, T., Braus, D. F., \& Buchel, C. (2006). Dissociable systems for gain- and loss-related value predictions and errors of prediction in the human brain. Journal of Neuroscience, 26, 9530-9537.

Yacubian, J., Sommer, T., Schroeder, K., Glascher, J., Kalisch, R., LEUENBERGER, B., ET AL. (2007). Gene-gene interaction associated with neural reward sensitivity. Proceedings of the National Academy of Sciences, 104, 8125-8130.

Yasuda, A., Sato, A., Miyawaki, K., Kumano, H., \& Kuboki, T. (2004). Error-related negativity reflects detection of negative reward prediction error. NeuroReport, 15, 2561-2565.

YeunG, N., \& SANFEY, A. G. (2004). Independent coding of reward magnitude and valence in the human brain. Journal of Neuroscience, 24, 6258-6264.

Zhou, Q. Y., \& Palmiter, R. D. (1995). Dopamine-deficient mice are severely hypoactive, adipsic, and aphagic. Cell, 83, 1197-1209.

(Manuscript received August 2, 2007; revision accepted for publication October 18, 2007.) 